\title{
B. Traven und Mexiko
}

\author{
Traditionen und Aktualität von Kapitalismuskritik \\ und Sozialutopie
}

\author{
Marc Hieger
}

\begin{abstract}
"Traven is getting modern. Reading Traven, is part of good form «, writes the German communist journal Linkskurve in 1932. Today largely forgotten, B. Traven, the voice of the Mexican Indians, at his time was an internationally acclaimed author. This article argues that he it is still worthwhile reading him. B. Traven's novels synthesize exotic flair, adventure, and social accusation. His descriptions of the social and political developments in Mexico can be related to the present in various ways. The article points out the timelessness of B. Traven's writing by focusing on the novel The Rebellion of the Hanged (Die Rebellion der Gehenkten; 1936). It tries to connect the literary fiction to recent developments in Mexico, e.g. to the drug war and to the continuous resistance of the EZLN. The utopia of a socially balanced, just and anti-racist society is a major subject in $B$. Traven's novel.
\end{abstract}

Title: B.Traven and Mexico: Tradition and Current Relevans of the Critik of Capitalism and Social Utopia

Keywords: B.Traven (1882?-1969?); Caoba-novels; critique of capitalism; exile literature, Mexican indians

Linke Sozialkritik, die in Form von Abenteuer- und Unterhaltungsliteratur den Leser anspricht, an ihn appelliert, ist heute nicht mehr en vogue. Schnell wird von ideologisch belasteter Literatur gesprochen, die seit dem Ende der DDR - dort Teil des nationalen Literaturkanons - zu Recht dem Vergessen anheimfiel. Beispielhaft dafür stehen all die Schriftsteller, die sich dem sozialistischen Realismus verpflichtet fühlten und heute per se verpönt sind. Ihre Werke gelten nicht nur als ideologisch verbrämt, sondern auch als unästhetisch und langweilig.

Es gibt aber auch andere, ebenfalls vergessene bzw. verdrängte linke Literaturtraditionen in Deutschland, die im anderen deutschen Staat akzeptiert waren, aber dennoch über jeden Zweifel der ideologischen Vereinnahmung bzw. Anbiederung erhaben sind. B. Traven, ${ }^{1}$ der bekannteste Unbekannte der deutschsprachigen Literatur, der mit wechselnden Pseudonymen und einem verheimlichten Lebenslauf alle biografischen Linien verwischen wollte, schrieb über Mexiko und seine jüngste Geschichte; er setzte sich mit den wirtschaftli-

1 Auch das am häufigsten genutzte Pseudonym B. Traven findet sich in den unterschiedlichsten Ausprägungen: Bruno, Ben oder auch Berick Traven [Torsvan]; vgl. dazu die ausführliche Darstellung von Ruiz Castañeda/Márquez Acevedo 2000: 819-821. 
chen, sozialen und politischen Umbrüchen dieses Landes auseinander. Seine Romane waren ab den $1950 e r$ Jahren in beiden deutschen Staaten echte Klassiker der Abenteuer- und Unterhaltungsliteratur und für den Leser in hohen Auflagen greifbar (in der »Sowjetisch-besetzten Zone« sind die ersten Roman [-auszüge] schon ab 1946 erhältlich [vgl. B. Traven 1946]). Eine intensivere wissenschaftliche Rezeption seiner Erzählungen und Romane setzte in den 1970 er Jahren sowohl in West- als auch in Ostdeutschland ein. Dennoch wurde dem Autor seine Kommunismus-Kritik in der DDR nicht immer verziehen, was eine temporäre Zensur einzelner Bücher zur Folge hatte (vgl. Hohnschopp 1989). Die wissenschaftliche Auseinandersetzung hält zwar bis heute an, versickert aber zunehmend; in den letzten Jahren sind nur noch vereinzelte Abhandlungen zu Autor und Werk erschienen. Ab den 1980er Jahren gingen die Auflagen seiner populärsten Romane und Erzählungen zurück, die Leser verloren B. Traven zunehmend aus den Augen.

Diese Entwicklung in Deutschland überrascht insofern, als die Gesamtauflage seiner Werke weltweit auf 30 Millionen Exemplare geschätzt wird (vg1. Jachmann 2011), er weiterhin gelesen und wahrgenommen wird. B. Traven ist in Mexiko nach wie vor ein bekannter und geachteter Autor, der zur nationalen Revolutions- und Indianerliteratur zählt (vgl. Rall/Rall 2000: 469; vgl. Ávila 2008). Er hat einen beachtlichen Einfluss auf mexikanische Autoren ausgeübt, die sich mit der Kultur und Lebenswelt der Chiapas-Indigenas beschäftigt haben (vgl. Rall 1996: 39). Dies ist umso mehr bemerkenswert, als die zentrale Diskussion, ob B. Traven überhaupt als mexikanischer (oder eben als deutscher) Autor anzusehen ist, seit Jahren geführt wird und weiter anhält (vgl. Rall 1996).

Am 26. März 1969 starb B. Traven in Mexiko-Stadt. Sein 40. Todestag hätte 2009 auch in Deutschland zu einem gesteigerten Interesse, zum Aufkommen einer Art >B.-Traven-Renaissance< führen können, aber dem war nicht so. Weder Verlage noch die germanistische bzw. literaturwissenschaftliche Forschung rückten diesen linkskritischen Autor wieder stärker in das öffentliche Bewusstsein. Mexiko-Stadt ehrt dagegen einen seiner bekanntesten Bürger bis heute und Malú Montes de Oca de Heyman, die Stieftochter B. Travens, äußerte im Jubiläumsjahr, sie wolle zukünftig in Deutschland neues Interesse für sein Werk wecken (vgl. Anonymus 2009). Die >B.-Traven-Abstinenz< ist nicht nur unverständlich, sondern ärgerlich, bieten sich doch heute in der mexikanischen Gesellschaft unübersehbare Anknüpfungspunkte, an denen der aktuelle - nicht nur politische - Gehalt und die Überzeitlichkeit seines Werkes aufgezeigt werden kann.

B. Traven rückte wie kein anderer Schriftsteller die indigene Bevölkerung Mexikos, ihre Situation und Lebensweise in das Bewusstsein einer breiten (in der zweiten Hälfte der $1920 e r$ Jahren zunächst deutschen) Leserschaft. ${ }^{2}$ Er stellte den besonders grausamen, unterdrückerischen Kapitalismus Mexikos dar, übte

2। Da die Begriffe Indianer oder Indio eine abwertende oder generalisierende Bedeutung beinhalten können, wird in dieser Darstellung auf ihre Verwendung (außer in Zitaten) verzichtet. 
in seinen Romanen und Erzählungen auch bissige Gesellschafts- und Systemkritik im Gewand unterhaltsamer, exotischer Abenteuer- und Unterhaltungsliteratur. Er gilt als einziger deutschsprachiger Schriftsteller des 20. Jahrhunderts, der sich in dieser Form der Welt der Maya-Quiché Indigenas in Chiapas angenähert hat. Es gibt nichts Vergleichbares, was dem Engagement, Verständnis und Wunsch B. Travens entspricht, diese außergewöhnlichen Kulturen und zugleich die Ausbeutung ihrer Menschen vor der Revolution zu beschreiben. Für andere Autoren sind die Chiapas-Indigenas in Abenteuerromanen eher ein geschichtliches Thema, ein dekoratives Moment, das höchstens ein sekundäres Motiv bildete (vgl. Rall 1996: 56f.). Nicht so im Werk von B. Traven, der als Nichtmexikaner zum vorausblickenden Beobachter des post-revolutionären Mexikos wurde. Geneigte Landsleute sehen in ihm einen der ihren, der mit großer Liebe und einem tiefen Verständnis den mexikanischen Charakter als auch die Indigenas im Südosten Mexikos verstanden habe (vgl. Carballo 2005). Denn in seinen Romanen und Erzählungen - seien sie auch mehr als 50 Jahre alt findet man die historischen und politischen Ursachen verarbeitet, mit denen man heute das Scheitern des großen mexikanischen Gesellschaftsprojektes, der Begründung einer neuen, gerechteren und multiethnischen Gesellschaftsordnung, erklären kann. An anderer Stelle heißt es zu B. Travens Erzählwerk: »Travens Caoba-Romane haben noch nichts von ihrer [politischen] Brisanz eingebüßt. Die zeitlose Grundproblematik der Romane und ihre kunstvolle literarische Verarbeitung vermögen auch heute noch Leser weltweit $\mathrm{zu}$ faszinieren.« (Brenne 2006: 330) Nicht zufällig fiel B. Travens Name in der mexikanischen Presse als einer der ersten, als sich 1994 die EZLN (Ejército Zapatista de Liberación Nacional [>Zapatistisches Heer der nationalen Befreiung‘]). erhob. Er galt immer noch als Synonym für den Kampf der Ureinwohner um gesellschaftliche und soziale Rechte; erneut wurde die Welt auf die politische und soziale Misere in seiner Wahlheimat Chiapas aufmerksam (vgl. Rall 1996: 57). Dass er »trotz seiner entschiedenen Kritik an kolonialistischer und neokolonialistischer Ausbeutung selbst noch in koloniale und gar rassistische Denkmuster verstrickt war«, hat Lürbke (2005: 95) untersucht. Aber sie stellt auch fest, dass »Traven der erste Schriftsteller überhaupt [war], der sich mit der Unterdrückung der Indigenas in Chiapas beschäftigte« (ebd.: 96).

Im Folgenden soll gezeigt werden, dass sich zentrale Beobachtungen und Feststellungen, wie sie sich in B. Travens Texten engagiert manifestieren, bis in die gesellschaftliche Gegenwart Mexikos reichen. Insbesondere seine Caoba-Romane stützen die Vision einer gerechteren, sozial ausbalancierten Gesellschaft. Dieser Aufsatz möchte dazu anregen, diesen schillernden und geheimnisvollen Autor wieder verstärkt zur Kenntnis zu nehmen, ihn als einen wichtigen, überzeitlichen Vertreter linkspolitischen Denkens zu würdigen.

Kurz vor Ende des Ersten Weltkriegs wurde B. Traven in München als amerikanischer Staatsbürger, geboren am 25. Februar 1882 in San Francisco, registriert. Später korrigierte der Autor in seinem Testament diese Angaben leicht: Er sei der 1890 in Chicago geborene Travens Torsvan Croves (vgl. Raasch 2006: 155). Kein deutschsprachiger Autor hat sich selbst derart zu einer lebenden Le- 
gende stilisiert: B. Traven (alias Traven Torsvan, B.T. Torsvan, Hal Croves [vgl. Brenne 2006: 25]) ist nach letzten Erkenntnissen jener ominöse Otto Feige, der in der heute polnischen Stadt Schwiebus zur Welt kam (vgl. Hauschild 2009: Z3). ${ }^{3}$ Er selbst bestritt zu Lebzeiten ausdrücklich deutscher Nationalität zu sein. ${ }^{4}$ Dies war eine lebenslange Strategie, die eigene Herkunft zu verschleiern, am besten ganz zu verwischen. Das Abwiegeln und Ablehnen einer definierbaren Identität und des eigenen Berühmtseins steigerte die Popularität, den Mythos »B. Traven« nur noch mehr.

\section{B. Traven alias Ret Marut 1923}

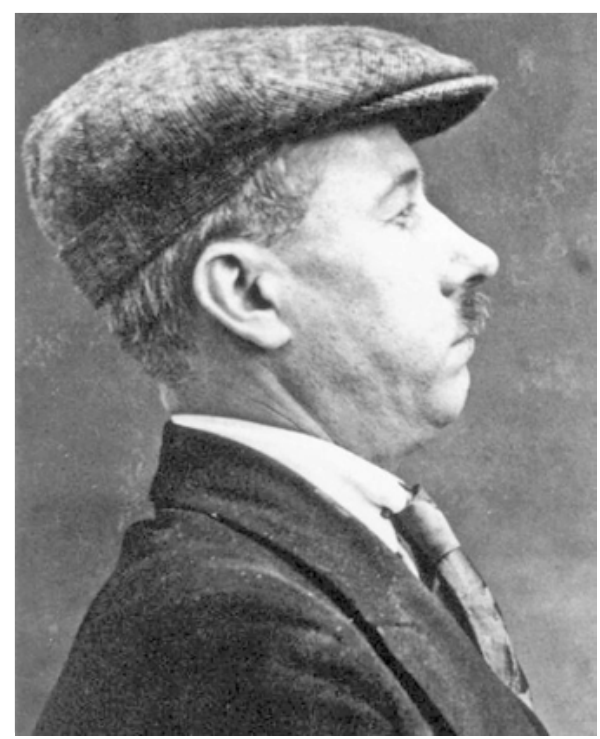

Zunächst begründete er als Ret Marut 1917 in München die anarchistische Zeitschrift Der Ziegelbrenner und wirkte 1919 als leitender Zensor in der Münchner Räterepublik (vgl. Brenne 2006: 20). ${ }^{5}$ Im Zuge des gewaltsamen Endes dieses rätedemokratischen Experiments wurden im selben Jahr dessen Führer durch Reichswehrtruppen verhaftet und erschossen. Aufgrund seines Engagements geriet B. Traven ebenfalls in deren Visier und konnte sich im letzten Moment der Verhaftung und Exekution durch seine Flucht nach Mexiko entziehen. Er ging zunächst in den Untergrund und floh in das europäische Ausland; die Details seiner Flucht liegen bis heute allerdings im Dunkeln (vgl. Guthke 1987:

3 | Die Recherchen des englischen Journalisten Will Wyatt mündeten in den 1970er Jahren in einer BBC-Dokumentation, die aber in der B.-Traven-Forschung lange Zeit keine große Beachtung fand.

4 | Vgl. B. Travens Brief vom 22. Mai 1938, der in der spanischen sozialistischen Tageszeitung Solidaridad Obrera abgedruckt wurde (vgl. Guthke 1987: 487), oder den Brief an John Schikowski vom 26. August 1925 (vgl. B. Traven/Schikowski 1992: 36). 5 | Zu B. Travens Biografie vgl. Recknagel 1982: 324f.; Guthke 1987; Hauschild 2012. 
33). 1924 kam B. Traven schließlich in Columbus, Tampico (Bundesstaat Tamaulipas), an und hielt sich zunächst mit Hilfsarbeiten auf Baumwollplantagen und Erdölfeldern über Wasser (vgl. Raasch 2006: 41). Ab Mitte desselben Jahres wurde er nach eigenen Angaben in einem Busch-Bungalow wieder schriftstellerisch tätig. Neben den eigenen Erfahrungen mit den hiesigen Lebens- und Arbeitsverhältnissen traten später seine Beobachtungen indigener Lebenswelten fernab städtischer Zivilisation, die Eingang in seine Texte fand. Im späteren Rückblick unterschieden sich diese - typisch für B. Travens humanen Standpunkt - nicht so sehr von denen der sog. zivilisierten Völker (vgl. Arenas 1976: 15). B. Traven beschäftigte sich intensiv mit der Kultur und Lebensweise indigener Völker in den vor-kolumbianischen Epochen, der Zeit der spanischen Eroberungen sowie der Gegenwart. Seine Erkenntnisse ließ er in seine wissenschaftlichen Abhandlungen und literarischen Texte einfließen (vgl. B. Traven 1968a, 1968c u. 1992). Damit eignete er sich ein fundiertes und breites Wissen an, welches ihm den Zugang zur Lebenswirklichkeit der Chiapas-Indigenas erleichterte. B. Traven bereiste 1926 das erste Mal für vier Monate Chiapas, den südlichsten mexikanischen Bundesstaat (Raasch 2006: 42f.). Zwei Jahre später erschien als Ergebnis dieser Eindrücke und Erfahrungen seine Reisebeschreibung Land des Frühlings (1928), die auch eigene Fotos enthält. Er sah darin auch eine landeskundliche, fundierte Analyse, die im (deutschsprachigen) Ausland Beifall fand; B. Traven wollte als sozialistischer, anarchistischer Autor nicht nur informieren und unterhalten, sondern auch politisch aufklären. Dieser Reiseund Abenteuerbericht, der soziologische und anthropologische Fragestellungen aufwirft und diese unter sozialkritischen Prämissen beantwortet, ist einer der zentralen Grundlagentexte für seinen späteren Caoba-Romanzyklus (vgl. Rall 1996: 71).

B. Traven wurde erst 1951 mexikanischer Staatsbürger. Zu diesem Zeitpunkt war er zum einen ein gefeierter Autor, der sich kenntnisreich und kritisch mit indigenen Kulturen auseinandergesetzt hatte und sich zweifelsohne als ihr Anwalt sah. Zum anderen wurde, aufgrund der radikalen linkspolitischen Tendenz seines Werkes, seine »mexikanische Authentizität« auch bezweifelt und angegriffen (Anonymus 1978b: 39). ${ }^{6}$ Bemerkenswert ist, dass B. Traven als Nichtmexikaner die miserable soziale Lage und Rechtlosigkeit der Indigenas thematisierte und als Gewissen einer Nation fungierte, welche sich bis heute nicht adäquat ihren gesellschaftlichen und politischen Problemen stellen kann, geschweige denn sich mit einem Teil ihrer kulturellen Identität ausgesöhnt hat. Auch heute gelten Indigenas in Chiapas immer noch als Menschen zweiter Klasse, die entrechtet leben und nach wie vor auf diese Situation aufmerksam machen (vgl. Anonymus 2012). B. Traven starb 1969 in Mexiko-Stadt und seine Asche wurde über dem Urwald von Chiapas verstreut, dem Haupthandlungsort seiner Caoba-Romane, die zu den wichtigsten seines Euvre zählen.

6 | Die von Januar 1951 bis April 1960 in Mexiko-Stadt und Zürich erschienenen B. Traven-Mitteilungen (BT-M) sind eine Rarität und wertvolle Quelle für die B.-TravenForschung; sie wurden als Information an Zeitschriften und Verlage verschickt. 
Die Romane und Erzählungen, die B. Travens schriftstellerischen Ruhm begründeten und ihn weltberühmt machten, erschienen in den Jahren 1924 bis 1930. Exotische Beschreibungen eines fernen Landes, die Lebensweisen der Menschen sowie die politischen Zustände vor, in und nach der mexikanischen Revolution (1910-1920) rückten Mexiko auch in Deutschland in das Blickfeld einer breiten Leserschaft. Sein wichtigstes Werk, der Caoba-Zyklus, auf dessen Roman Die Rebellion der Gehenkten (1936) im Folgenden genauer eingegangen werden soll, ist, ungeachtet seiner formalen Mängel, »zur großen historischen und kritischen Romanliteratur unseres Jahrhunderts zu rechnen « (Pogorzelski 1985: 280). Der Zeitgenosse Kurt Tucholsky (1989: 304) äußerte mit gewohnter Schärfe, aber auch anerkennend: »Ein großer Epiker. Sicherlich kein sehr angenehmer Herr, sicherlich kein sehr glücklicher Mensch. Aber ein großer Epiker.«

Die Romane des Caoba-Zyklus: Der Karren (1930), Regierung (1931), Der Marsch ins Reich der Caoba (1933), Die Troza (1936), Die Rebellion der Gehenkten und Ein General kommt aus dem Dschungel (1940), verorten ihre Handlungen alle im südlichen Bundesstaat Chiapas und weisen Verbindungslinien untereinander auf (z.B. treten dieselben Figuren auf, der Leser kann ihre Lebenswege mitverfolgen). ${ }^{7}$ Der Autor spannt dabei einen zeitlichen Bogen von den letzten Jahren der Herrschaft von Porfirio Díaz ${ }^{8}$ bis zur mexikanischen Revolution. Das spanische Wort »Caoba« bedeutet »Mahagoni«. Das der Romanzyklus nach dieser Baumart benannt wurde, lässt den Leser schon im Vorfeld die wirtschaftliche Bedeutsamkeit dieses Rohstoffes, seiner Industrie und die sozialen und politischen Konsequenzen für die Arbeitermassen in den Urwäldern erahnen. Alle Romane haben die Ausbeutung und Unterdrückung der indigenen Bevölkerung in Chiapas zum Gegenstand, welche bis zu ihrer Erhebung mit staatlicher Duldung bis zum Äußersten ausgebeutet wird. B. Traven erschafft ein engagiertes sozial-politisches Gemälde dieser Zeit, dieser Romanzyklus ist »Abrechnung, Rechtfertigung, Botschaft und Testament zugleich« (Brenne 2006: 37). Nach wie vor gilt die Aussage, dass die »Caoba-Romane dem Leser die verschiedensten Bereiche der gesellschaftlichen, kulturellen und historischen Realität« Mexikos erschließen (Pogorzelski 1985: 271).

B. Travens Caoba-Roman Die Rebellion der Gehenkten gilt als sein politisch wirkungsvollstes Buch, da sich die Öffentlichkeit über Jahrzehnte immer wieder damit auseinandergesetzt hat, es verfilmt und auch auf die Theaterbühne ge-

7 | Der vollständige Zyklus ist online unter http://nemesis.marxists.org/traven-dercaoba-zyklus.htm [Stand: 30.06.2013] einsehbar.

8 | Porfirio Díaz (1830-1915), mexikanischer General und Politiker, wurde 1876 mit Unterstützung des Militärs Staatspräsident und regierte diktatorisch zunächst bis 1880, ein zweites Mal war er von 1884 bis 1911 im Amt. Der "Porfirismo" forcierte mit US-amerikanischem und britischem Kapital die Industrialisierung und Modernisierung (Eisenbahn) des Landes. Die Masse der Indigenas partizipierte weder in wirtschaftlicher noch sozialer Hinsicht an dieser Entwicklung. 
bracht wurde (vgl. Raasch 2006: 97). ${ }^{9}$ Die überzeitliche Wirkungsabsicht des Buches wird bereits in den BT-Mitteilungen 1951 angedeutet:

Es ist wiederum ein Appell an das Menschheitsgewissen, im übertragenen Sinne sogar eine beispielhafte Drohung, wie Traven den Aufstand der versklavten indigenen Plantagenarbeiter im mexikanischen Urwald gegen ihre weißen Unterdrücker bis zur staatserschütternden Revolution entwickelt. Traven will nicht nur spannend unterhalten. Er stellt seine unerhörte Meisterschaft in den Dienst eines Kampfes für soziale Gerechtigkeit. (Anonymus 1978a: 35)

Die Überzeitlichkeit des Romans zeigt sich in der Abbildung politischer Strukturen oder Mechanismen, die bis in die Gegenwart existieren und deren (Aus-) Wirkungen andauern. B. Traven will realistisch und auf geschichtlichen Fakten basierend erzählen, dennoch sind seine Bücher nicht als historische Romane oder gar Dokumente zu lesen. Seine Texte haben eine aufklärerische Intention, die zur »unmittelbare[n] Parteinahme« (Bergmann 1976: 268) des Lesers führen soll.

Im Roman Die Rebellion der Gehenkten wird der Leser in die menschenfeindliche Wildnis der Monterias geführt, in riesige Gebiete des tropischen Regenwaldes. Die Handlung erstreckt sich von 1910 bis 1911, zeigt also die Anfänge der mexikanischen Revolution. Dort wird in großen Holzfällerlagern Mahagoniholz für ausländische (spanische oder auch nordamerikanische) Unternehmen geschlagen. Eine der Hauptfiguren des Romans ist der Tsotil-Indigena ${ }^{10}$ Candido $^{11}$ Castro, ein armer, aber unabhängiger Kleinbauer. Seine kranke Frau fällt einem Arzt in Jovel (San Cristóbal de Las Casas) zum Opfer. Sie muss sterben, da der »Doktor « zunächst auf sein Geld für die Behandlung wartet und ihr nicht hilft. Dem moralisch intakten Mikrokosmos der indigenen Siedlung »Rancheria«, aus der Candido stammt, wird die unmoralische, verkommene Welt der Städter Jovels gegenübergestellt. Der Arzt verlangt Geld für nicht durchgeführte Untersuchungen, und der Bestatter verdient ebenfalls am Tod der Frau. Am Ende ist der Protagonist hoch verschuldet und gerät mit seinen Kindern und seiner

9 | Vgl. u.a. die Verfilmung von Alfredo B. Crevenna u. Emilio Fernández (Regie): La rebelión de los colgados (Spielfilm). Mexiko 1954 (85 Min.); mit Pedro Armendáriz, Aridna Welter und Carlos López Moctezuma. B. Traven schrieb unter dem Pseudonym Hal Croves gemeinsam mit John Bright am Drehbuch, und Gabriel Figueroa führte die Kamera. 1986 folgte noch eine dreiteilige TV-Produktion der Pidax film media Ltd.; Regie führt der Franzose Juan Luis Buñuel, das Drehbuch bearbeitete Hans W. Geissendörfer; zu den Mitwirkenden gehörten Gustav Maria Halmer ('Don Ramon`), Uwe Ochsenknecht ('Franzose`) und Reiner Schöne.

10 | Die Tsotsil- oder Tzotzil-Maya bewohnten ursprünglich die südlichen Gebiete von Chiapas, die an der Grenze von Guatemala liegen. Die Maya-Kultur breitete sich über die gesamte Yucatán-Halbinsel aus, d.h. umfasste Landesteile, die heute zu Mexiko, Guatemala, Honduras und El Salvador gehören.

11 | Candido (span.): (der) Treuherzige, Naive; in Anlehnung an Voltaires philosophische Erzählung Candide (1759). 
Schwester Modesta in die Fänge des Agenten Don Gabriel, der mit Besitzern der Holzplantagen zusammenarbeitet und für sie billige Arbeitskräfte rekrutiert. Die Minen- als auch Großgrundbesitzer sind bei ihrer Suche nach billigen Arbeitskräften stets bestrebt, unabhängige Kleinbauern (»Peones«) und Arbeiter mit Knebelverträgen zu fangen und in ihren Dienst zu pressen. Die den Indigenas eigene Vertrauensseligkeit und Höflichkeit wird ihnen im Roman immer wieder zum Verhängnis. Die Ladinos können stets mit ihrer Unterwürfigkeit und ihrem Gehorsam rechnen, die Mestizen belügen, unterdrücken und beuten die Indigenas aus und fürchten niemals irgendeinen Aufruhr. Der Erzähler zeichnet aus indigenen Blickwinkeln ein übertrieben negatives, fast chauvinistisches Bild der Ladinos, um zugleich die tiefe Menschlichkeit der Indigenas zu zeigen, aber auch um ihre Apathie, ihren Fatalismus anzuprangern. Candido befindet sich von nun an in einem rechtlosen, quasi sklavischen Arbeits- und Abhängigkeitsverhältnis. Im Urwald soll er seine Geldschuld auf einer Holzplantage abarbeiten. Trotz aller mündlichen Zusagen und Versprechungen wird er auf Jahre ein Gefangener der Holzgesellschaft und ihrer Besitzer sein. Es bleibt ungewiss, ob er oder seine Familie überleben werden.

Auf die kulturellen Gemeinsamkeiten und tiefen Widersprüchlichkeiten zwischen den unterschiedlichen Bevölkerungsteilen (Kreolen, Mestizen, Indigenas) in Neu-Spanien hat Octavio Paz 1979 in einem Essay hingewiesen: »Wie der Kreole ist der Mestize weder Spanier noch Indio; er ist auch kein Europäer, der die Verwurzelung sucht [...]. Seine Beziehung zu den Indigenas gehorcht derselben Ambivalenz: er ist ihr Henker und ihr Rächer.« (Paz 1984: 30) In Das Labyrinth der Einsamkeit schreibt er 1950, dass die Unterdrückung der Indigenas und damit der vorkolumbianischen Kultur mit der Suche des Mexikaners nach seiner Herkunft, seinen Ursprüngen zusammenfalle (Paz 1998: 29). Und weiter heißt es:

Der Mexikaner will weder Indio noch Spanier sein; ebenso wenig will er von innen abstammen. Er verleugnet sie, und er behauptet weniger, ein Mestize zu sein als dessen Abstraktion: ein Mensch. Er möchte von niemanden abstammen, seinen Ursprung bei sich selber nehmen. (Ebd.: 90)

Ganz im Gegensatz dazu stand bereits das Konzept politischer Emanzipation, das Benito Juárez ${ }^{12}$ als erster indigener Präsident Mexikos verfolgte. Er wollte einen neuen, ethisch-moralischen und auf Recht basierenden Staat begründen, der »eine universelle, abstrakte Auffassung vom Menschen proklamierte: >Die Republik besteht demnach nicht aus Kreolen, Indios und Mestizen [...], sondern einfach aus Menschen - und nur aus solchen.« (Paz 1998: 91) Die freie und unabhängige mexikanische Gesellschaft sollte sich nur auf die Gleichheit aller Menschen vor dem Gesetz begründen (ebd.: 127). ${ }^{13}$ Leider blieb seine gro-

12 | Benito Juárez (1806-1872), liberaler Politiker indigener Abstammung, übernahm 1858 die Regierung und wurde 1861 Präsident von Mexiko.

13 IIm Gegensatz zu anderen lateinamerikanischen Ländern, in denen heute immer noch große indigene Bevölkerungsgruppen und Mehrheiten existieren, gingen diese im 
ße politische Vision unvollendet, denn bis heute bilden die Mestizen die Oberschicht der mexikanischen Gesellschaft, die sich rigide und auch chauvinistisch von der indigenen Bevölkerung abgrenzt. Aus einem »kolonialen Patchwork « (Baxmann 2007: 152) resultiert eine tiefgreifende Störung der mexikanischen Identität, von den Anfängen der spanischen Kolonisation bis in die Gegenwart.

Auch José Vasconcelos ${ }^{14}$ versuchte zu Beginn des 20. Jahrhunderts das Identitätsproblem der mexikanischen Gesellschaft zu lösen. In seinen Schriften setzt er sich mit der indigenen Kultur auseinander, würdigt ihre ethnologische Bedeutung und versucht die Indigenas in die sozialen Hierarchien seines Landes einzugliedern (vgl. Krumpel 2006: 46). Dies bedeutete jedoch auch die Verwerfung indigener Kultur(en) (vgl. Sauter de Maihold 1995: 71ff.). Nach der mexikanischen Revolution leistet Vasconcelos mit seinen Werken La raza cósmica (1925) und Indología - Una interpretación de la cultura ibero-americana (1927) der Idee der »Mestizaje«, d.h. der Mischung indianischer und abendländischer Kulturen, Vorschub. Er bezieht sich dabei auf die »ethnische Homogenität« der indigenen Völker während der vorkolumbianischen Epochen, mit der er die Verbindung abendländischer und indigener Kultur propagiert (vgl. Vasconcelos 1925: 19). Diese ist im Prinzip die logische Konsequenz der jahrhundertealten Mischung der Bevölkerungsteile Mexikos und sie wird nach der Revolution lange zur nationalen Identitätsformel, der »Mexicanidad« (Rössner 2007: 264). Die heutige mexikanische Gesellschaft offenbart aber das Scheitern dieser »Mexicanidad«. Zum einen wird indigene Kultur auf nationaler Ebene erinnert, geschätzt und gefördert (man denkt sofort an die berühmten Wandgemälde Diego Riveras), zum anderen ist ihr gegenüber ein latenter Rassismus in der mexikanischen Gesellschaft verankert, der die soziale, rechtliche und politische Ungleichheit der Indigenas weiter festschreibt. ${ }^{15}$

In B. Travens Roman repräsentieren tyrannische Charaktere wie die Montallo-Brüder das gescheiterte Konzept der »Mexicanidad«. Die Holzgroßplantagenbesitzer gehören zu den neuen aufstrebenden Kapitalisten Mexikos. Die von ihnen zu verantwortenden Arbeitsbedingungen und Lebensumstände im

Verlauf der mexikanischen Geschichte hauptsächlich in der "Mestizo-Kultur" auf. Etwa $90 \%$ der Mexikaner haben heute sowohl europäische als auch indigene Vorfahren. Vom Nationalen Indigenen Institut (INI) wird die Größe der Urbevölkerung heute auf 10,6 Millionen Menschen geschätzt, die sich auf 56 verschiedene "pueblos indios" (indigene Völker) verteilen (vgl. Ross 2004: 34).

14 | José Vasconcelos (1882-1959) wurde 1914 unter Präsident Obregón Erziehungsminister und beeinflusste maßgeblich die mexikanische Kulturpolitik. Er versuchte durch zahlreiche Maßnahmen, wie Lehrmittelfreiheit, Alphabetisierungskampagnen oder den Aufbau von Schulen und Bibliotheken, die Bildungssituation indigener Bevölkerungsgruppen zu verbessern.

15 | Nach UN-Kriterien leben die als sehr arm eingestuften 18 Millionen Mexikaner zumeist in jenen 803 Landkreisen, die als "indigen " ausgewiesen sind. Die Kindersterblichkeit ist in diesen um ein Vielfaches höher und die Lebenserwartung (von Erwachsenen), Alphabetisierungsrate und Einkommen liegen deutlich unter dem der nicht-indigenen Bevölkerungsgruppen im Land (vgl. Ross 2004: 35). 
Urwald fordern jeden Tag ihre Opfer. Alle Arbeiter müssen mindestens zwei Tonnen Holz pro Tag schlagen. Da es aber keine nachvollziehbaren, vertraglichen Abmachungen gibt, ist diese Vorgabe rein willkürlich:

[Candido:] "Zwei Tonnen, Patroncito, so steht es in meinem Kontrakt. [...]"

[Don Felix:] "Was in deinem Kontrakt steht, geht uns hier gar nichts an, und du hältst dein stinkiges Maul, oder ich lasse gleich einen Capataz [Aufseher] rufen, um dir den Montería-Willkommensgruß zu geben. [...] Drei Tonnen Lieferung den Tag, und schaffst du keine drei Tonnen, wird dir der Tag nicht bezahlt; und wenn das einige Tage so faul weitergeht, wirst du ja sehen, wie wir dich zu drei Tonnen herankriegen." (B. Traven 1936: 58)

Auch das Aufeinandertürmen der großen »Trozas« (Holzstöße), die den Fluss hinuntergeflößt werden, kostet Menschenleben. Die ausbleibende Entlohnung ist die geringste Strafe für »schlechte Arbeit«, üblich ist das »Henken«, falls nicht genügend Tonnen Holz pro Tag herangeschafft werden. Die Schreie der Bestraften hallen als Warnung durch den Urwald, dabei werden die Opfer, an Händen und Füßen gefesselt, an Bäumen heraufgezogen:

Auf dem Boden lagen acht Klumpen Mensch. Sie waren völlig in sich zusammengequetscht, als wären sie für Monate in engen runden Fässern aufbewahrt gewesen. [...] Die Lassos, an denen die Klumpen hingen, waren mit einigen geübten Griffen der Capataces aufgeknotet worden, und man hatte die Körper einfach zu Boden fallen lassen, ohne sich weiter darum zu kümmern. [...] Ob sie während des Henkens verreckten, wie es zuweilen geschah, oder ob sie nicht verreckten, hatte für die Montellanos und deren Aufseher nur insofern Interesse, als innen Arbeitshände verlorengingen. Wenn die Hände zu faul sein sollten und nicht fähig waren, drei oder vier Tonnen zu schaffen, so war nichts verloren, wenn der Mann verreckte. (Ebd.: 73)

Diese unmenschlichen Strafen lösen schließlich die »Rebellion der Gehenkten« aus. Willkür und Unmenschlichkeit regieren in den Monterias, ein Menschenleben zählt dort nichts, Geldgier und Profitmaximierung dagegen alles. Betroffen sind zwar auch weiße Arbeiter, aber vor allem doch die Indigenas, was die rassistische Komponente dieser Ausbeutung verdeutlicht.

Die Zerstörung indigener Gemeinschaften, die auf landwirtschaftlicher Produktion basieren, sind nach marxistischen Überlegungen Voraussetzung kapitalistischen Wirtschaftens:

In allen naturalwirtschaftlichen Formationen - ob es sich um primitive Bauerngemeinden mit Gemeindeeigentum an Grund und Boden, feudale Fronverhältnisse oder dergleichen handelt - ist die Produktion für den Selbstbedarf das Ausschlaggebende der Wirtschaft [...]. [Sie] stützen sich in ihrer wirtschaftlichen Organisation auf die Fesselung des wichtigsten Produktionsmittels - des Grund und Bodens - sowie der Arbeitskräfte durch Recht und Herkommen. Die Naturalwirtschaft setzt somit den Bedürfnissen des Kapitals starre Schranken entgegen. Der Kapitalismus führt deshalb vor allem 
stets und überall einen Vernichtungskampf gegen die Naturalwirtschaft ..., so in auBereuropäischen Ländern - im Kampfe gegen primitivere soziale Formen - in der Gestalt der Kolonialpolitik. (Luxemburg 1985: 316f.)

Der nordamerikanische Kapitalismus strebt in Mexiko danach, diese »starren Schranken« zu durchbrechen. Marx (2009: 717) spricht von der »Vernichtung des auf eigener Arbeit beruhenden Privateigentums, d.h. [...] [der] Enteignung des Arbeiters.« Die Modernisierung Mexikos unter Díaz, d.h. die Errichtung von Verkehrswegen (z.B. der Eisenbahn) sowie Bergbau- und anderen Industriezentren, war wichtig für den Aufbau eines vorindustriellen Kapitalismus. Voraussetzung dafür waren hohe Auslandsinvestitionen, die den mexikanischen Staat in äußere Abhängigkeiten brachte. Die Entwicklung und der Ausbau von Wirtschaft und Infrastruktur gehorchten folglich den Interessen des ausländischen Kapitals. Der »Porfirismo« sah sich selbst in einer liberalen Tradition; dennoch verlängerte er, wie es Paz (1998: 129f.) beschreibt, einen »anachronistischen, rücksichtlosen Feudalismus«. Immerhin hätten die »Indianer-Gesetze« schon damals Vorschriften zum Schutz der Indigenas enthalten. Der Landbesitz gehörte nun nicht mehr der Kirche, sondern der besitzenden mexikanischen Aristokratie und damit wieder einer kleinen, immer mächtiger werdenden Minderheit. Unter dem Díaz-Regime wurde im Zuge des Auf- und Ausbaus der Infrastruktur, so bei der Landvermessung und der Errichtung von Eisenbahnlinien, etwa ein Drittel des öffentlich klassifizierten Bodens privaten Gesellschaften zugesprochen und viele Kleinbauern und Viehzüchter somit entschädigungslos enteignet (vgl. Bernecker/Pietschmann /Tobler 2007: 228). Die Enteignungen von indigenem Grundbesitz führten dazu, dass die wirtschaftliche Unabhängigkeit der Indigenas beendet wurde. Rosa Luxemburg (1985: 319) formuliert dies so:

Die gewaltsame Methode ist die [...] direkte Folge des Zusammenpralls des Kapitalismus mit naturalwirtschaftlichen Formationen [...] [er] strebt zielbewusst danach, sie als selbständige soziale Gebilde zu vernichten.

Im Kampf gegen naturalwirtschaftliche Gesellschaften oder Formationen folge der Kapitalismus ökonomischen Prämissen, so z.B. »sich wichtiger Quellen von Produktivkräften direkt zu bemächtigen, wie Grund und Boden, [...] Erzeugnissen exotischer Pflanzenwelt [...] und [...] Arbeitskräfte >frei< zu machen und zur Arbeit für das Kapital zu zwingen.« (Ebd.: 317f.)

Die Ausbeutung indigener Arbeiter und die unmenschlichen und lebensgefährlichen Arbeitsbedingungen auf den Holzplantagen, die B. Traven in seinem Roman beschreibt, sind Teil dieses Akkumulationsprozesses. Ausländische Firmen bauten mit Hilfe mexikanischer Unternehmer das Mahagoniholz billig ab und exportierten es nach Europa (vor allem Großbritannien) oder in die USA. Damit zeigt B. Traven in seinem Roman auch die beginnende weltwirtschaftliche Abhängigkeit Mexikos und dessen Einbindung in das im 19. Jahrhundert entstehende global-kapitalistische Wirtschaftssystem. Die Besitzer der Monteri- 
as lassen das Mahagoniholz rücksichtslos schlagen, und - parallel zur Vernichtung der Menschen - wird das Land auch ökologisch zerstört.

In B. Travens Roman repräsentiert der Staat eine institutionalisierte Ungerechtigkeit, der unterprivilegierte Bevölkerungsmehrheiten unterdrückt und deren ökonomische Ausbeutung forciert. Die Regierung und ihre Institutionen sind Instrumente in den Händen der Besitzenden. Durch Gouverneure oder Staatsbeamte in der Verwaltung und Justiz wird das freie Zusammenleben in den unabhängigen Dorfgemeinschaften (»pueblos«) untergraben und geschwächt, um Kleinbauern in die »peonaje « (Schuldknechtschaft) zu treiben. ${ }^{16}$ Im Roman werden Militär und Polizei als besonders korrupte und skrupellose Kasten charakterisiert, die Autorität und Macht stets für sich nutzen. Unter dem Díaz-Regime werden die modern und technisch überlegen ausgerüsteten Truppen der »rurales« (Landgendarmerie) aufgestellt, die Aufstände blutig niederschlagen und durch Gewalt und Terror bestehende politische Verhältnisse aufrechterhalten:

Wenn fünfzig Rurales auf fünfhundert aufständische indianische Landarbeiter losgingen, dann hatten am Ende der Schlacht die Rurales vielleicht drei Tote und keinen Verwundete, während die Rebellen vierhundertsechzig Tote und keinen Verwundeten auf dem Schlachtfelde zurückließen. (B. Traven 1936: 221)

Die zu Beginn des Romans auftretenden Charaktere, wie der örtliche Polizeikommandant oder der Arzt, sind Repräsentanten des korrupten Staates. Die Großgrundbesitzer oder auch die Agenten wie Don Gabriel können sich auf die Unterstützung der Behörden verlassen, mit denen man sich stets »geschäftlich « und gütlich einigt. Die Stadtbewohner und ihre Handelsaktivitäten sind von der Kaufkraft und Produktivität der Indigenas abhängig, die dennoch mit der größten Geringschätzung behandelt werden. Im Gegensatz zur Stadt wird an anarchistische Ideen angelehnt - die dörfliche indigene Kommune in den Caoba-Romanen zum Idealbild einer funktionierenden Lebensgemeinschaft stilisiert, die sich von der Verderbtheit der »mexikanischen« bzw. »europäischen« Zivilisation und damit der Stadtkultur abgrenzt. In ihr lasse sich noch indigene Kultur mit ihren integralen Bestandteilen von Gleichheit und Gerechtigkeit realisieren: Hier »tritt dem Leser die gesellschaftliche Praxis der mexikanischen Mayas im Sinne einer >konkreten Utopieく entgegen« (Pogorzelski 1985: 276). Die ärmlichen, aber unabhängigen Dörfer und Siedlungen der Indios bilden Räume der Freiheit im Gegensatz zu den »Fincas« der Großgrundbesitzer, die ebenfalls danach trachten, die Ureinwohner mit legalen oder illegalen Mitteln in ihre Dienste zu zwingen. Diese Gemeinschaften, die traditionell auf starken familiären und kommunalen Bindungen basieren und nur so existieren kön-

16 | B. Traven zeichnet in seiner Erzählung Diplomaten (1930) ein ironisches Bild des vor-revolutionären Mexikos, das an eine politische Analyse und Bestandsaufnahme erinnert (vgl. B. Traven 1968b). 
nen, bilden einen diametralen Gegensatz zur ausbeuterischen und materiellen Welt der Finceros, Städter oder Holzplantagenbesitzer:

Obgleich das Land der übrigen Familien um nichts besser war als das des Candido Castro, obgleich alle in sehr ärmlichen Palmhütten, spärlich mit Lehm beworfen, wohnten [...], so war es nicht nur gegenüber Candido, sondern auch gegenüber allen anderen Familien nie geglückt, eine von ihnen nach einer Finca als Peones hinwegzulocken. Es wusste ein jeder Mann in der Siedlung, dass ein Leben der Peones auf den Fincas in vieler Hinsicht leichter, sicherer und zuverlässiger war als das auf dieser mageren, heißen, dürren, steinigen Erde. [...] Jedoch alle die Familien zogen vor, lieber ärmlich zu leben, ewig bedroht von verlorenen Ernten, als sich unter Herrschaft und Kommando zu begeben [...]. (B. Traven 1936: 8)

Urbano, ein Leidensgenosse Candidos, denkt beim Anblick des Flusses, in dem die Trozas abgeschwemmt werden, an die Gegenden, »wo das Wasser hinziehe, [in denen] wohl Frieden sein müsse und dort wahrscheinlich schöne Dörfer an den Ufern lägen, wo Menschen wohnten - Menschen mit guten Herzen und mit Liebe zu allen ihren Nachbarn« (ebd.: 96).

Paz stellt dazu fest, dass die indigenen Landarbeiter sich der mexikanischen Revolution deshalb anschlossen, um ihr Land zurückzugewinnen (Paz 1998: 140f.). Die Möglichkeit einer autonomen Versorgung stammt aus vorkolumbianischen Zeiten und war bereits unter der Herrschaft der Mayas etabliert. Hunger und Verelendung, die sich im Zuge der spanischen Eroberung seit dem 16. Jahrhundert unter der Urbevölkerung ausbreiteten und bis in das 19. Jahrhundert anhielten, waren bis dato unbekannt. Durch die Reformgesetzgebung im 19. Jahrhundert wurde das Gemeindeeigentum fast gänzlich aufgelöst und abgeschafft. Die Folge davon war eine vertiefte Abhängigkeit von den Großgrundbesitzern, die aus dieser Politik gestärkt hervorgingen (ebd.: 141). ${ }^{17}$ Während der mexikanischen Revolution erließ Präsident Venustiano Carranza unter dem Druck der Zapata-Bewegung (benannt nach Emiliano Zapata ${ }^{18}$ ) 1915 ein Gesetz, welches die Rückgabe der »ejidos« (Gemeindeländereien) regeln

17 | Im Zuge einer liberalen Reformära (1855/56) wurde die Enteignung kirchlichen Landbesitzes umgesetzt und eine Verfassung (1857) eingeführt. In dieser waren Wahlrecht, Grundrechte, Bildungsfreiheit und eine freie Wirtschaft vorgesehen. Die Ley Lerdo (1856) sah auch den Verkauf des seit alters her unveräußerlichen Kommunallandes der Indigenas vor. Damit sollte die mexikanische Wirtschaft zusätzlich modernisiert und neu belebt werden, den indigenen Kommunen drohte allerdings die gänzliche Verelendung. Es handelte sich letztendlich um eine entschädigungslose Enteignung (vgl. Bernecker u.a. 2007: 153f.).

18 | Emiliano Zapata (1883-1919), mexikanischer Revolutionär, spielte insbesondere als Anführer der Bauern ab 1906 eine herausragende Rolle in der Revolution. Die Zapata-Bewegung enteignete in inrem Herrschaftsbereich (südlicher Teil des Hochlandes, Stadt Morelos) jeglichen Großgrundbesitz und stellte das Land freien Kleinbauern zur Verfügung (vgl. Tobler 1992: 185). Die indigene Befreiungsbewegung der Zapatisten (EZLN) im Bundesstaat Chiapas beruft sich heute noch auf inn. 
sollte. Während seiner Präsidentschaft wurde ebenfalls die konzeptionell sehr fortschrittliche Verfassung von 1917 ausgearbeitet und in Kraft gesetzt, die mit den Artikeln 27 und 123 - sie »definieren das Privateigentum als Schöpfung des Staates gemäß dem Gemeininteresse - den Landarbeitern und damit der breiten Masse der Indigenas das Recht auf Boden garantieren sollte (vgl. Rey 1976: 287). Carranza und seine Regierung wollten damit die Masse der Landarbeiter für sich gewinnen, die im Süden des Landes vor allem auf Seiten der Revolutionäre Zapata und Franciso »Pancho« Villa (1894-1923) standen (vgl. Beck u.a. 1976: 280). ${ }^{19}$ Paz unterstreicht die gesellschaftliche Bedeutung des indigenen Gemein- und Kollektivbesitzes:

Indem [...] [der Radikalismus der mexikanischen Revolution] aus dem Calpulli das Grundelement unserer wirtschaftlichen und sozialen Organisation schuf, hat der Zapatismus nicht nur die Werte kolonialer Tradition gerettet, sondern auch bestätigt, daß jede wirklich fruchtbare Konstruktion von dem ältesten, stabilsten und dauerhaftesten Kern unserer Nation ausgehen sollte: der indianischen Vergangenheit. (1998: 143)

Er betont, dass während der Regierungszeit von Lázaro Cárdenas die »institutionalisierte Revolution « (ebd.: 152) versucht habe, sich mit größerer Breiten- und Tiefenwirkung in der Gesellschaft durchzusetzen. B. Traven verfasste seinen Roman während dieser Präsidentschaft. Cárdenas versuchte mit seiner Politik den traditionellen Gemein- und Kollektivbesitz neu zu beleben. Ein zentrales Ziel seiner tiefgreifenden Sozialreformen war die Lösung der Agrarfrage durch die Enteignung von Großgrundbesitz und dessen Verteilung an landlose Kleinbauern. Diese erlebte 1936/37 ihren Höhepunkt und 1940 umfassten die »ejidos « 47,4\% des gesamten Ackerlandes in Mexiko (vgl. Bernecker u.a. 2007: 296). Das Projekt war in einen »Pakt« eingebunden: Die nachrevolutionären Regierungen garantierten dem Volk Bildung, Arbeit und Stabilität, aber keine Demokratie. Solange diese Übereinkunft aufrechterhalten wurde, war Mexiko so charakterisiert es Carlos Fuentes (2004: 253) - bis zu den großen Unruhen 1968 das lateinamerikanische Modell für Stabilität. Noch in der Ära der seit dem Ende der mexikanischen Revolution regierenden Staatspartei Partido de la Revolución Institucional (PRI; >Partei der institutionalisierten Revolution<) wurde in den $1990 e r$ Jahren der verfassungsrechtliche Rahmen so geändert, dass die bis dato stattfindende Landverteilung nicht nur gestoppt, sondern Kommunalland nun auch privatisiert werden konnte. ${ }^{20}$ Der visionären Politik Lázaro Cárdenas', in der sich mit der Landverteilung an unabhängige Kleinbauern das Hauptziel der mexikanischen Revolution zu erfüllen schien, wurde endgültig das Grab

19 | Das von Zapata als Oberbefehlshaber unterzeichnete Manifest an das mexikanische Volk zeigt, dass man den neuen Herrschern der Republik und ihrer Politik großes Misstrauen entgegenbrachte. Eine ernsthafte Umsetzung, d.h. die Enteignung des Großgrundbesitzes und dessen Umverteilung, wurde nach Meinung der Revolutionäre niemals in Erwägung gezogen (vgl. Zapata 2005: 140f.).

20 | Zur Änderung des Art. 27 der mexikanischen Verfassung vgl. Horn 2004: $125 f$. Vgl. auch Werner 1992: 11. 
geschaufelt. In Chiapas haben diese großflächigen Privatisierungen bis in die Gegenwart zu einer massiven Beeinträchtigung der Umwelt geführt, die wiederum die Existenz der dort lebenden Indigenas gefährdet. ${ }^{21}$

B. Traven schildert in Die Rebellion der Gehenkten den Beginn einer hoffnungsvollen, aber auch brutalen Revolution, an deren Ende ein funktionierendes Gemeinwesen stehen könnte, das, gerade mit Blick auf die Ejido-Genossenschaften, an die Verwirklichung einer anarchistischen Gemeinschaft erinnert. Die freie indigene Kommune, so wie sie B. Traven in dem zweiten Roman des Caoba-Zyklus Regierung (1931) anhand der Siedlung »Solipaz« (»sol y paz«: >Sonne und Frieden $<$ ) beschreibt, weist bereits in Richtung einer gesamtgesellschaftlichen Utopie und ist eine »Metapher einer herrschaftsfreien Gesellschaft « (Hohnschopp 1993: 232). Allerdings schließt der Zyklus mit Ein General kommt aus dem Dschungel (1940) ab, der einen offenen Schluss aufweist und damit für den Leser ebenfalls offen lässt, ob die (zapatistische) Revolution scheitern oder erfolgreich sein wird.

Carlos Fuentes äußert mit Blick auf die heutigen Verhältnisse, dass die mexikanische Revolution Chiapas nie erreicht habe, es statt dessen dort »eine Plutokratie, eine Oligarchie [gibt], die Land und Volk ausbeutet und von Banditen, Handlangern, Pistolenhelden und auch von den lokalen Strukturen der [...] PRI [...] unterstützt werde« (Fuentes/Barloewen 2003: 256). Zu Beginn der 199oer Jahre wiederholte sich in Chiapas die mexikanische Revolution in einem kleineren Format. Allerdings ging es den Aufständischen des Zapatistischen Nationalen Befreiungsheeres (EZLN) nicht darum, militärische Siege zu erringen. Der Aufstand war eine Machtdemonstration und die Schaffung eines Machtfaktors, mit dem die Regierung rechnen und verhandeln musste, insbesondere was die Reaktivierung und Verteidigung des Gemeindelandes anbelangte. Subcomandante Marcos machte zu Beginn des Jahres 1994 als Anführer und Sprecher der EZLN-Guerilleros die Welt auf die Situation der Indigenas aufmerksam. ${ }^{22}$ Schwer bewaffnet besetzten die Aufständischen zunächst die zweitgrößte Stadt im südmexikanischen Bundesstaat Chiapas, San Cristóbal de las Casas. Die Indigenas hatten bereits jahrelang vergeblich mit legalen Mitteln für die Anerkennung ihrer Autonomierechte, den Schutz ihres Lebensraumes und gegen ihre Diskriminierung gekämpft. ${ }^{23} \mathrm{Nach}$ wie vor sind Großgrundbesitz, alte und neue Formen von Sklaverei, Analphabetismus sowie ausufernde Staatskorruption allgegenwärtig und verbreitet. Ein wesentlicher Auslöser des Aufstandes der EZLN war auch der Beitritt Mexikos zum Nordamerikanischen Freihandels-

21 Die anhaltende massive Ausplünderung von Rohstoffen und Bodenschätzen (u.a. gibt es in Chiapas große Gas- und Ölfelder der staatlichen Ölgesellschaft PEMEX) führt zur Zerstörung des Ökosystems. Nur im geringen Umfang werden Profite in der Region reinvestiert (z.B. in die Gesundheitsversorgung, Bildung, Industrie oder Infrastrukturprogramme); vgl. dazu Subcomandante Insurgente Marcos 2005.

22 | Vgl. insbes. EZLN/Ya Basta [2005]. Zur Entwicklung der Guerilla und ihres Aufstandes siehe Kerkeling 2006.

23 | Vgl. dazu u.a. Tena 1994: 3; Gabbert 2004; Zimmering 2005; vgl. auch Bernecker u.a. 2007: $356 f$. 
abkommen (NAFTA) im Januar 1994, das für die Masse indigener Kleinbauern zur existentiellen Bedrohung wurde. ${ }^{24}$ Die Privatisierung von Gemeindeland ist seitdem verfassungsrechtlich abgesichert und beschleunigt dessen Ausverkauf an ausländisches Kapital, was wiederum die Selbst- und Grundversorgung der Landbevölkerung stark gefährdet. Die mexikanische Regierung ging im weiteren Verlauf der Auseinandersetzungen massiv gegen die Aufständischen der EZLN und die indigene Bevölkerung vor, es kam zu Bombardierungen mit vielen Toten. Nach einem Waffenstillstand folgten jahrelange Verhandlungen. Die 1996 erzielte Vereinbarung (das Abkommen von San Andrés ${ }^{25}$ ) zur Verbesserung der rechtlichen Situation der Indigenas und dem Schutz ihrer Kultur wurde bis heute nicht umfassend umgesetzt.

In B. Travens Die Rebellion der Gehenkten ist die Ermordung brutaler Aufseher der Auslöser des Aufstandes gegen die Caoba-Herren, und eine gewaltsame, unaufhaltsame Kettenreaktion wird in Gang gesetzt. Der ganze Hass trifft nun die Besitzer der Holzplantagen, die an Ort und Stelle wie gefährliche Tiere totgeschlagen werden. Die Indigenas werden militärisch organisiert und von erfahrenen Rebellen angeführt. Mit dem Ruf »Tierra y Libertad« (>Erde und Freiheit $<$ machen sich die Holzarbeiter in großen Kolonnen auf den Weg, die Revolution aus dem Urwald in das gesamte Land zu tragen. Der Erzähler kommentiert, dass Zensur, Unterdrückung der Gedankenfreiheit sowie Bildungsmangel in sinnlose, destruktive Gewalt münden können:

Es war nicht die Schuld der Rebellen, daß sie zerstörungswütig und mordlustig waren. Nie hatten sie sich besprechen können, nie hatten sie sich beraten dürfen, nie kam jemand, der sie in politischen oder in wirtschaftlichen Dingen unterrichtete. Keine Zeitung durfte die Politik des Diktators kritisieren. Kein Buch gab es, das Arbeiter gelehrt hätte, wie sich ihre Lage verbessern ließe, ohne Mord und ohne Zerstörung. (B. Traven 1936: 206f.)

Der Überfall auf die Hacienda am Ende des Romans, die nicht zufällig »El Requemado« (span.: >versengt<, >verbrannt<, >verkohlt<) heißt, wirft auch ein zweifelhaftes Licht auf die beginnende Revolution. B. Traven stellt damit die Frage, ob sich der Aufstand als konstruktiv, gesellschaftsumwälzend und letzten Endes als die Erfüllung einer gesellschaftlichen Utopie erweisen wird oder in Destruk-

24 Durch die Einfuhr von billigem Importmais aus den USA wurden bis heute bis zu 700000 Bauern in den Ruin getrieben (vgl. Raasch 2006: 128-131; Ross 2004: 109114). Im Februar 2008 forderten Tausende Bauern in Mexiko-Stadt Neuverhandlungen über das Freihandelsabkommen mit den USA und Kanada. Amerikanische Landwirte werden mit Subventionsgeldern mit jährlich bis zu 20000 Dollar gefördert, während ein mexikanischer Kleinbauer höchstens 700 Dollar im Jahr erhält (vgl. Anonymus 2008: 7).

25 | Die drei Grundsatzdokumente legten die Verfassungsreformen fest, die die Autonomie der indigenen Völker in Mexiko garantieren sollten. Hier wurden auch Möglichkeiten nicht-institutioneller politischer Organisation, Beteiligung und Selbstverwaltung eingeräumt (vgl. Hernández Navarro/Vera Herrera 1998). 
tion und Terror endet. Die in der Nähe der Hacienda lebenden Bauern töten den Verwalter und seine Familie und übernehmen die Besitzung. Sie schließen sich nicht den revolutionären indigenen Arbeitern an.

B. Travens Kritik und Skepsis gegenüber der Revolution bzw. ihrem möglichen Scheitern am Ende des Romanzyklus weist ebenfalls in die Gegenwart. Es stellt sich heute die Frage, inwieweit die Indigenas im heutigen Mexiko ökonomisch und politisch gleichberechtigt leben können. In den 1990er Jahren weitete sich der EZLN-Aufstand zu einem regelrechten Krieg aus, in dem viele Menschen ihr Leben ließen, Verwüstung und Zerstörung die Folge waren. Der Konflikt schwelt weiter, unübersehbar ist, dass die ungelösten politischen und sozialen Probleme nach wie vor einen brisanten gesellschaftlichen Sprengstoff darstellen. Das basisdemokratische und kollektive Denken der indigenen Kommunen ermöglichte in Oaxaca einen friedlichen Widerstand, der erst durch das gewalttätige Vorgehen der Staatsmacht eskalierte. Nach den Zusammenstößen waren 20 Tote und Hunderte von Verletzten zu beklagen. Einige von der Polizei Festgenommene sind seitdem verschwunden. ${ }^{26}$ Die mexikanische Zeitung $L a$ Jornada urteilte am 13. August 2007:

Die Leute aus Oaxaca haben dagegen viel Erfahrung mit einer anderen Form der Demokratie: mit jener, die auf radikale Weise in den indigenen Dörfern praktiziert wird und auch ein substantielles soziales Gewebe in den Städten bildet, wie sich kürzlich an den Barrikaden gezeigt hat. [...] Es hat den Anschein, dass sie heute den Widerstand in einen Befreiungskampf umwandeln, der diesen sozialen und politischen Lebensstil auf den gesamten Staat ausdehnen soll - und zwar jenseits der herrschenden, bloß formalen Demokratie. (Zit. n. Mercker 2007: 1184)

Die freie indigene Kommune als nicht-korrupte, kollektive Alternative, wie sie auch von B. Traven dargestellt wird, hat insofern einen Modellcharakter, da sie basisdemokratisch organisiert ist und ihren Bewohnern wirtschaftliche Autonomie und Sicherheit bietet. Die Anerkennung und Wertschätzung indigener (politischer) Kultur und Tradition seitens des Staates wäre nicht nur eine versöhnende Geste, sondern könnte auch nachhaltig die Entwicklung einer gerechten, sozialen Zivilgesellschaft unterstützen. Carlos Fuentes stellt im historischen Rückblick fest, dass die große mexikanische Revolution nicht nur einen enormen gesellschaftlichen, sondern auch einen kulturellen Bewusstwerdungsprozess in Gang gesetzt habe (vgl. Fuentes/Barloewen 2003: 268). Er fragt: »Verdienen wir ein anderes Schicksal?« Und antwortet sogleich: »Es muss unsere Antwort sein!«

Die Indigenas Mexikos sind Teil unserer multikulturellen und multiethnischen Gesellschaft. Sie zu vergessen, heißt uns selbst zum Vergessen zu verurteilen. Die Gerechtigkeit, die sie erhalten, sollte untrennbar von der sein, die wir selbst erhalten. Die

26 | Das brutale Vorgehen von 5000 Bundespolizisten in Oaxaca von Juli bis Dezember 2006 zeigt, dass der Staat auf die virulenten gesellschaftlichen und politischen Probleme in Mexikos Süden häufig gewalttätig reagiert (vgl. dazu Mercker 2007). 
mexikanischen Indigenas sind das Zünglein an der Waage unserer gemeinschaftlichen Gesellschafts-Möglichkeit. Wir werden niemals zufriedene Männer und Frauen sein, wenn wir nicht das Brot mit innen teilen. (Fuentes 1989: 20f.)

B. Travens Texte lassen nicht nur Bezüge zu aktuellen politischen Themen und Entwicklungsmustern zu, sondern verweisen auch auf mögliche Perspektiven einer gerechteren und sozial ausbalancierten Gesellschaft. Er würdigt die indigene Traditionen des gesellschaftlichen und wirtschaftlichen Zusammenlebens und versuchte bereits vor über 50 Jahren zu ergründen, warum indigene Lebens- und Kulturtraditionen für die mexikanische Gesellschaft der Gegenwart und Zukunft unverzichtbar sind. Den freien Menschen sieht er wie Emiliano Zapata im freien, selbstbestimmten Indigena, der eine Nation würdiger Menschen begründet. Die im Roman Die Rebellion der Gehenkten enthaltene Kapitalismuskritik erscheint aktueller denn je. Die konservative Regierungspraxis hat mit ihrer Wirtschaftspolitik in den vergangenen Jahren suggeriert, dass in nicht kontrollierten Märkten ein Allheilmittel für die wirtschaftlichen und sozialen Probleme des Landes zu sehen sei. Damit erteilen sie dem indigenen Konzept des Gemein- und Kollektivbesitzes eine Absage - mit furchtbaren Folgen. Für B. Traven galt die dörfliche indigene Kommune als Keimzelle einer freiheitlichen Gesellschaft, in der sich das selbstbestimmte, politisch emanzipierte Individuum entfalten kann. Seit 2003 haben die Chiapas-Indigenas zunächst mit Hilfe der EZLN regionale Selbstverwaltungsstrukturen etabliert. In kleinen und politisch autonomen Einheiten, den sogenannten Caracoles, wird seitdem in alter Mayatradition versucht, eigene lokale Bildungs- und Gesundheitsprogramme aufzubauen (vgl. Matamoros 2006: 16). Kleine Betriebe, Geschäfte und Kooperativen werden gefördert und jegliche Unterstützung durch die Bundesregierung wird abgelehnt. Ein funktionierendes Beispiel ist der biologische und kooperative Kaffeeanbau (vgl. dazu Gerber 2005). Im Bundesstaat Oaxaca wurde - trotz der Auseinandersetzungen vor sechs Jahren - bereits 1998 das bislang fortschrittlichste Gesetz hinsichtlich indigener Rechte und Selbstverwaltung verabschiedet. Es räumt weitgehende Autonomie (einschließlich des Rechtes Steuern zu erheben) und kulturelle Selbstbestimmung ein. Carlos Fuentes, in dessen Erzählwerk die Revolution und das indigene Erbe eine zentrale Rolle spielen, bezieht sich immer wieder kritisch auf die heroische Vergangenheit Mexikos:

Juárez und Cárdenas waren groß, weil sie Männer ihrer Zeit mit historischem Gedächtnis waren. Wir können uns nicht aus Furcht vor der jetzigen Welt (die viel Erschreckendes hat) in die Sehnsucht nach unwiederholbaren Heldentaten flüchten. Wir sollten besser unsere Lektion lernen und Fehler vermeiden. (Fuentes 2004: 193)

Nationale Identität statuarisch festzulegen hieße, sie zu musealisieren. Die indigene Geschichte und Welt wird von ihren Erben als moderne Welt begriffen, die Respekt und Schutz verdient, »aber keine unkritische Schmeichelei, die ihr Elend, ihre Unwissenheit und ihre Ungerechtigkeit nur in alle Ewigkeit fortsetzt.« Fuentes stellt die Frage: »Werden wir im einundzwanzigsten Jahrhun- 
dert ein Land sein, das weder Angst vor seiner indigenen Vergangenheit noch vor seiner mestizischen Moderne hat?« (Ebd.: 192) Er sieht die einzige Option einer zukunftsweisenden Politik darin, die Ureinwohner Mexikos endlich in Wort und Tat als mündige Bürger anzuerkennen, die Rechte haben und am politischen Prozess teilnehmen müssen. Autonomie und Wohlstand der indigenen Regionen von Chiapas und ganz Mexiko hingen entscheidend von der Achtung kommunaler Freiheiten ab (vgl. Fuentes/Barloewen 2003: 256).

B. Traven hat den Ureinwohnern in Chiapas, stellvertretend für alle mexikanischen Indigenas, eine Stimme gegeben, die weit über die Landesgrenzen hinweg gehört wurde. Das Recht jedes Individuums und jedes Volkes, seinen eignen freien Weg zu gehen, schließt Unterdrückung, Nötigung und Dogmatismus aus. Angesichts der zunehmenden Gewalt in Mexiko, die aus dem in den letzten Jahren verschärften Anti-Drogenkampf der Regierung resultiert, stellt sich gegenwärtig die Frage nach der Autorität staatlicher Institutionen und ob sie die Gesellschaft überhaupt noch vor der weiter eskalierenden Gewalt beschützen können (vgl. dazu Ehringfeld 2008: 9). Die Ursachen sind wirtschaftlichen und sozialen Ursprungs, der Drogenkrieg ist zum wesentlichen Teil Ergebnis der Verelendung breiter Bevölkerungsmassen. ${ }^{27}$ Mexikos neuer Präsident Enrique Peña Nieto hat einen Strategiewechsel im Anti-Drogenkrieg angekündigt, in dem der Schutz der Bevölkerung und nicht die Vernichtung der Drogen-Bosse Priorität hat. Ob er erfolgreich sein wird, bleibt abzuwarten (vgl. Ehringfeld 2012: 9).

B. Traven zeigt in seinem Caoba-Zyklus und im Roman Die Rebellion der Gehenkten sozialutopische Ansätze auf. Die indigene Kommune kann Ausgangspunkt eines umfassenden humanen, nachhaltigen Denkens und Handelns sein. Für ihn stehen nicht bestimmte politische Programme oder Ideologien im Vordergrund, sondern er spricht allgemein von der Humanisierung, dem Finden von unterschiedlichen Meinungen und Lösungen (vgl. Árenas 1976: 14). B. Traven versteht sich als ein Suchender, der die Ursprünge menschlicher Humanität aus sich heraus vermitteln möchte, wie er zwei Jahre vor seinem Tod in einem Interview erläutert:

Der Schriftsteller, ein sich seiner Menschlichkeit bewußter Mensch, betrachtet, zu diesen Ursprüngen zu gelangen, nicht als Befehl, Verpflichtung oder "Aufgabe«. Für den Schriftsteller ist es etwas Selbstverständliches zu diesen Ursprüngen zu gelangen, obwohl es nicht so einfach wie essen, beobachten oder zu Fuß gehen ist. (Ebd.: 14f.)

Ein Autor muss humanistisches Denken leben, es ist ihm Verpflichtung, die ihn zu seiner Tätigkeit berechtigt: Der »Schriftsteller, der nicht zum Ursprung geht, ist kein Schriftsteller« (ebd.: 14) Auf die Frage, ob ein Autor eher ein Kämpfer oder ein passiver Betrachter dessen sei, was ihn umgebe, antwortet B. Traven:

27 | Dieser Krieg wurde immer blutiger, brutaler und hat seit Dezember 2006 28000 Tote gefordert, mit steigender Tendenz (vgl. dazu Keppeler 2010). 
Von der tiefen Beobachtung aus, wenn man in ihr die wesentliche Innerlichkeit der Realität sucht, daraus entsteht eine kämpferische Haltung dem Leben gegenüber und das Streben nach einem besseren Leben. Das Leben ist ein Kampf. Wenn man innen [den Lesern] den Kontrast des ganzen Lebens zeigt, mit seiner Schönheit, Fruchtbarkeit und seiner Lebensfreude, bekämpft man die Ungerechtigkeit, die Kleinmütigkeit, die Unehre, die Diskriminierung, die Unterwürfigkeit. Die echte Literatur schließt die rein passive Beobachtung aus. (Ebd.)

Literatur muss seiner Ansicht nach einen humanen, kämpferischen Standpunkt einnehmen. Das Aufzeigen von Formen kapitalistischer Ausbeutung, die sich in ihrer grundsätzlichen Struktur am Beispiel des heutigen Mexikos weiterhin konkretisiert, liegt den Romanen B. Travens zugrunde. Carlos Fuentes beschreibt Mexiko »oxymoronisch« als ein Land der Widersprüche, das »sanfte Stärke, grausames Mitleid, tödliche Freundschaft, Leben im Augenblick [ist]. Alle Zeiten sind in einer vereint, die Vergangenheit jetzt, die Zukunft jetzt, die Gegenwart jetzt.«(Fuentes 2004: 195). B. Traven ist Fuentes »Kronzeuge«, wenn er ebenfalls die Bedeutung indigener Lebensweisen und Traditionen hervorhebt und in kollektiven Lebensformen eine Perspektive für die Befriedung und nachhaltigen Entwicklung großer Teile der mexikanischen Gesellschaft sieht. Hierin zeigt sich vor allem der aktuelle Gehalt und die Überzeitlichkeit von B. Travens Werk. Im Mexiko der Gegenwart gilt er immer noch als Romancier der Zärtlichkeit, Bescheidenheit, Aufrichtigkeit und der Liebe (vgl. Carballo 2005), auch deshalb hat sein Werk wieder größere Beachtung sowohl in Deutschland als auch Mexiko verdient.

\section{LITERATUR}

Anonymus (1951a): Die Rebellion der Gehenkten. In: Esperanza Lopez Mateos/Rosa Elena Lujan/Josef Wieder (Hg.): B. Traven-Mitteilungen (BT-M), No. 1-36. Mit einem Vorwort (Leitfaden zur Handhabung der BT-Mitteilungen) von Kilian Schott [ND]. Berlin (West) 1978, S. 35

Anonymus (1951b): Was Mexiko über Traven schreibt. In: Esperanza Lopez Mateos/ Rosa Elena Lujan/Josef Wieder (Hg.): B. Traven-Mitteilungen, No. 1-36. Mit einem Vorwort von Kilian Schott [ND]. Berlin (West) 1978, S. 39.

Anonymus (2007) in: La Jornada v. 13. August 2007), Ulrich Mercker: Mexiko: Oaxaca probt den Aufstand. In: Blätter für deutsche und internationale Politik 52, Nr. 10, S. 1184.

Anonymus (2008): Bauernproteste in Mexiko. In: Frankfurter Allgemeine Zeitung, Nr. 28 v. 2. Februar 2008 , S. 7.

Anonymus (2009): El misterioso Traven está presente en México. In: El Universal v. 13. September 2009; online unter: http://www.eluniversal.com.mx/cultura/ 60482.html [Stand: 30.06.2013]. 
Anonymus (2012): Demonstration in Mexiko. Zapatisten fordern Freiheit. In: taz. die tageszeitung v. 22. Dezember 2012; online unter: http://www.taz.de/Demonstrationin-Mexiko/ !107952 [Stand: 30.06.2013].

Arenas, José (1976): Nuestro Traven. In: Plural VI, Nr. 61, S. 13-15.

Ávila, Wilfrido: Bruno Traven, personaje misterioso del cuento Mexicano. In: El Sol de Cuernavaca v. 18. April 2008; online unter: http://www.oem.com.mx/esto/notas/ n666912.htm [Stand: 30.06.2013].

B. Traven (1928): Land des Frühlings. Berlin.

Ders. (1936): Die Rebellion der Gehenkten. Zürich/Prag.

Ders. (1946): Karrenreise durch die Sierra Madre: Aus dem Roman "Der Karren«. Berlin/ Leipzig.

Ders. (1968a): Die Geburt eines Gottes [1928]. In: Ders.: Erzählungen. Erster Band. Frankfurt a.M./Wien/Zürich, S. 31-38.

Ders. (1968b): Diplomaten [1930]. In: Ders.: Erzählungen. Erster Band. Frankfurt a.M./ Wien/Zürich, S. 140-164.

Ders. (1968c): Sonnen-Schöpfung. Indianische Legende [1936]. In: Ders.: Erzählungen. Erster Band. Frankfurt a.M./Wien/Zürich, S. 317-347.

Ders. (1992): Himmel und Hölle der Azteken. In: Ders./John Schikowski: Ich kenne das Leben in Mexiko: Briefe an John Schikowski 1925 bis 1932. Mit einem Essay von Karl S. Guthke. Frankfurt a.M./Berlin, S. 28-32.

Ders./Schikowski, John (1992): Ich kenne das Leben in Mexiko: Briefe an John Schikowski 1925 bis 1932. Mit einem Essay von Karl S. Guthke. Frankfurt a.M./Berlin.

Baxmann, Inge (2007): Die transnationale Nation. In: Dies.: Mayas, Pochos und Chicanos. Die transnationale Nation. München, S. 152-158.

Bergmann, Klaus (1976): Kleine Geschichte der mexikanischen Revolution. In: Johannes Beck/ders./Heiner Boehncke (Hg.): Das B. Traven-Buch. Reinbek b. Hamburg, S. 268-283.

Bernecker, Walther L./Pietschmann, Horst/Tobler, Hans Werner (Hg.; 2007): Eine kleine Geschichte Mexikos. Frankfurt a.M.

Brenne, Hans Rudolf (2006): Revolution und Abenteuer. Struktur und Botschaft von B. Travens Caoba-Zyklus als eine Folge operativer historischer Romane. Beschreibung einer innovativen Romanform. Münster.

Carballo, Emmanuel (2005): Traven y las letras mexicanas. In: El Universal v. 27. April 2005; online unter: http://www.eluniversal.com.mx/columnas/47625.html [Stand: 30.06.2013].

Ehringfeld, Klaus (2008): Entfesselte Drogenkartelle. Mexikos Regierung erfolglos im Kampf gegen Mafia / UN-Experte fordert Strategiewechsel. In: Frankfurter Rundschau, Nr. 221 v. 21 . September 2008, S. 9.

Ders. (2012): Gezielte Schläge statt Krieg. Mexikos neuer Präsident Peña Nieto will neue Wege im Kampf gegen die Drogenkartelle gehen. In: Frankfurter Rundschau, Nr. 297 v. 20. Dezember 2012, S. 9.

EZLN/Ya Basta - Netz für Solidarität und Rebellion (Hg.; [2005]): EZLN. Sechste Erklärung aus dem lakadonischen Urwald. Lich.

Fuentes, Carlos (1989): Prólogo. In: Fernando Benítez: Los indios de México. Antología. México, S. 11-21.

Ders. (2004): Woran ich glaube. Alphabet des Lebens. München. 
Ders./Barloewen v., Constantin (2003): Kreolische Odyssee. Das Drama Lateinamerikas und die Mythologien der Zukunft. Ein Interview mit Carlos Fuentes. In: Barbara Dröscher/Carlos Rincón (Hg.): Carlos Fuentes' Welten. Kritische Reflexionen. Berlin, S. 253-284.

Gabbert, Wolfgang (2004): Der Aufstand der Zapatisten in Chiapas 1994. Vorbedingungen und Folgen. In: Walther L. Bernecker u.a. (Hg.): Mexiko heute. Politik - Wirtschaft - Kultur. 3. vollst. neu bearb. Aufl. Frankfurt a.M., S. 363-384.

Gerber, Philipp (2005): Das Aroma der Rebellion. Zapatistischer Kaffee, indigener Aufstand und autonome Kooperativen in Chiapas, Mexiko. Münster.

Guthke, Karl S.(1987): B. Traven. Biographie eines Rätsels. Frankfurt/OIten/Wien.

Hauschild, Jan-Christoph (2009): Wer ist dieser Mann? In: Frankfurter Allgemeine Zeitung, Nr. 140 v. 20. Juni 2009, Z3.

Ders. (2012): B. Traven - Die unbekannten Jahre. Zürich.

Hernández Navarro, Luis/Vera Herrera, Ramón (Hg.; 1998): Acuerdos de San Andrés. México.

Hohnschopp, Christine (1989): Der ,Eigene، im Prokrustesbett des Marxismus. Über die Traven-Rezeption in der DDR. In: Text+Kritik, H. 102, S. 50-59.

Dies. (1993): Rebellierende Tote: Tod und Emanzipationsprozess im Werk B. Travens. Paderborn.

Horn, Hans-Rudolph (2004): Aktualisierung der Verfassung von 1917. In: Walther L. Bernecker u.a. (Hg.): Mexiko heute. Politik - Wirtschaft - Kultur. 3. vollst. neu bearb. Aufl. Frankfurt a.M., S. 117-147.

Jachmann, Sven (2011): Comicbiografie eines Phantoms. Der Mann, der sich verbarg. In: taz. die tageszeitung v. 23. November 2011; online unter: http://www.taz.de/ Comicbiografie-eines-Phantoms/!82362 [Stand: 30.06.2013].

Keppeler, Toni: Eskalierende Gewalt. 28.000 Tote in Mexikos Drogenkrieg. In: taz. die tageszeitung v. 4. August 2010; online unter: http://www.taz.de/!56551 [Stand: 30.06.2013].

Kerkeling, Lutz (2006): ¡La lucha sigue! - Der Kampf geht weiter. EZLN - Ursachen und Entwicklungen des zapatistischen Aufstands. 2., akt. u. erweit. Aufl. Münster.

Krumpel, Heinz (2006): Philosophie und Literatur in Lateinamerika - 20. Jahrhundert. Ein Beitrag zur Identität, Vergleich und Wechselwirkung zwischen lateinamerikanischem und europäischem Denken. Frankfurt a.M. u.a.

Lürbke, Anna (2005): Die indigenen Kulturen Mexikos im Werk von B. Traven. In: Günter Dammann (Hg.): B. Travens Erzählwerk in der Konstellation von Sprachen und Kulturen. Würzburg, S. 95-108.

Luxemburg, Rosa (1985): Die Akkumulation des Kapitals. Ein Beitrag zur ökonomischen Erklärung des Imperialismus [1913]. In: Dies.: Gesammelte Werke. Bd. 5: Ökonomische Schriften. Berlin (Ost), S. 5-411.

Marx, Karl (2009): Das Kapital. Kritik der politischen Ökonomie. Erstes Buch: Der Produktionsprozess des Kapitals. Ungekürzte Ausgabe nach der zweiten Auflage von 1872. ND der Ausg. Berlin 1932. Köln 2009, S. 707-717.

Matamoros Ponce, Fernando (2006): Der Comandante und der Bürgermeister. In: Le Monde Diplomatique. Beilage der taz. die tageszeitung v. 10. Februar 2006, S. 16.

Mercker, Ulrich (2007): Mexiko: Oaxaca probt den Aufstand. In: Blätter für deutsche und internationale Politik 52, Nr. 10, S. 1180-1184. 
Paz, Octavio (1984): Quetzalcóatl und Tonantzín. In: Ders.: Essays I. Frankfurt a.M., S. $17-64$.

Ders. (1998): Das Labyrinth der Einsamkeit. Essay. Frankfurt a.M.

Pogorzelski, Winfried (1985): Aufklärung im Spätwerk B. Travens. Eine Untersuchung zu Inhalt, formaler Struktur und Wirkungsabsicht des Caoba-Zyklus. Frankfurt a.M./ Bern/New York.

Raasch, Rolf (2006): B. Traven und Mexiko. Ein Anarchist im Land des Frühlings: Eine politisch-literarische Reise. Berlin.

Rall, Dietrich (1995): B. Traven, ein mexikanischer Autor? In: Renate von Hanffstengel/ Cecilia Tercero Vasconcelos/Silke Wehner Franco (Hg.): Mexiko, das wohltemperierte Exil. Mexiko-Stadt 1995, S. 93-102.

Ders. (1996): Literatura y etnología: Los indios de Chiapas como tema en la narrativa Alemana y Mexicana. In: Ders./Marlene Rall (Hg.): Letras comunicantes. Estudios de literatura comparada. Mexiko-Stadt 1996, S. 21-82.

Ders./Rall, Marlene (2000): Zur Rezeption der deutschsprachigen Literatur in Mexiko. In: Manfred Durzack/Beate Landenberg (Hg.): Literatur im interkulturellen Dialog. Festschrift zum 60. Geburtstag von Hans-Christoph Graf v. Nayhaus. Bern u.a., S. $460-472$.

Recknagel, Rolf (1982): B. Traven. Beiträge zur Biographie. 3. Aufl. Leipzig.

Rey, Romeo: Die verlorene Revolution. In: Johannes Beck/Klaus Bergmann/Heiner Boehncke (Hg.): Das B. Traven-Buch. Reinbek b. Hamburg, S. 284-297.

Rössner, Michael (Hg.; 2007): Lateinamerikanische Literaturgeschichte. 3. erweit. Aufl. Stuttgart/Weimar.

Ross, John (2004): Mexiko. Geschichte - Gesellschaft - Kultur. Münster.

Ruiz Castañeda, María del Carmen/Márquez Acevedo, Sergio: Diccionario de seudónimos, anagramas, iniciales y otros alias usados por escritores mexicanos y extranjeros que han publicado en México. México 2000, S. 819-821.

Sauter de Maihold, Rosa María (1995): Del silencio a la palabra: mythische und symbolische Wege zur Identität in den Erzählungen von Carlos Fuentes. Frankfurt a.M. u.a.

Subcomandante Insurgente Marcos (2005): Chiapas: Der Südosten in zwei Winden, einem Sturm und einer Prophezeiung. In: Ders.: Botschaften aus dem lakandonischen Urwald. Hamburg, S. 18-40.

Tena, Gerado (1994): "Gerechter Krieg" gegen die Erben der Eroberer. In: taz. die tageszeitung v. 4. Januar 1994, S. 3.

Tobler, Hans Werner (1992): Die mexikanische Revolution. Gesellschaftlicher Wandel und Umbruch 1876-1940. Frankfurt a.M.

Tucholsky, Kurt (1989): B. Traven [1930]. In: Ders.: Gesammelte Werke. Hg. v. Mary Gerold Tucholsky u. Fritz J. Raddatz. Bd. 8. Reinbeck b. Hamburg, S. 296-304.

Vasconcelos, José (1925): Indología. Una interpretación de la cultura ibero-americana. Barcelona.

Werner, Johannes: Dem Kapital "Tierra y Libertad". Mexikos Bauerngenossenschaften, Erbe der Revolution von 1917, werden abgeschafft. In: taz. die tageszeitung v. 13. Mai 1992, S. 11.

Zapata, Emiliano (2005): Manifest an das mexikanische Volk [1916]. In: Ricardo Magón Flores: Tierra y Libertad. Hg. v. der Gruppe B.A.S.T.A. Münster, S. 137-147.

Zimmering, Raina (2005): Die Revolte gegen die "Revolution “, der Zapatismus. In: Dies. (Hg.): Der Revolutionsmythos in Mexiko. Würzburg, S. 150-164. 
